\title{
Dieldrin Augments mTOR Signaling and Regulates Genes Associated with Cardiovascular Disease in the Adult Zebrafish Heart (Danio rerio) $)^{\mathbf{S}}$
}

\author{
Logan Slade, Andrew Cowie, Chris J. Martyniuk, Petra C. Kienesberger, \\ and Thomas Pulinilkunnil
}

Department of Biochemistry and Molecular Biology, Dalhousie University, Dalhousie Medicine New Brunswick (DMNB), Saint
John, New Brunswick, Canada (L.S., A.C., P.C.K., T.P.); and Department of Physiological Sciences and Center for Environmental
and Human Toxicology, UF Genetics Institute. University of Florida, College of Veterinary Medicine, Gainesville, Florida (C.J.M.)

Received December 29, 2016; accepted April 5, 2017

\section{ABSTRACT}

Dieldrin is a legacy organochlorine pesticide that is persistent in the environment, despite being discontinued from use in North America since the 1970s. Some epidemiologic studies suggest that exposure to dieldrin is associated with increased risks of neurodegenerative disease and breast cancer by inducing inflammatory responses in tissues as well as oxidative stress. However, the direct effects of organochlorine pesticides on the heart have not been adequately addressed to date given that these chemicals are detectable in human serum and are environmentally persistent; thus, individuals may show latent adverse effects in the cardiovascular system due to long-term, low-dose exposure over time. Our objective was to determine whether low-level exposure to dieldrin at an environmentally relevant dose results in aberrant molecular signaling in the vertebrate heart. Using transcriptomic profiling and immunoblotting, we determined the global gene and targeted protein expression response to dieldrin treatment and show that dieldrin affects gene networks in the heart that are associated with processes related to cardiovascular disease, specifically cardiac arrest and ventricular fibrillation. We report that genes regulating inflammatory responses, a significant risk factor for cardiovascular disease, are upregulated by dieldrin whereas transcripts related to lysosomal function are significantly downregulated. To verify these findings, proteins in these pathways were examined with immunoblotting, and our results demonstrate that dieldrin constitutively activates Akt/mTOR signaling and downregulates lysosomal genes, participating in autophagy. Our data demonstrate that dieldrin induces genes associated with cardiovascular dysfunction and compromised lysosomal physiology, thereby identifying a novel mechanism for pesticideinduced cardiotoxicity.

\section{Introduction}

The widespread use of pesticides negatively impacts our environment, thereby rendering human health susceptible to chronic diseases, such as obesity, cancer, and neurodegeneration (Alavanja et al., 2004; Lee et al., 2014). Dieldrin, an organochlorine pesticide (OCP), is a persistent chemical that was banned from agricultural use in the 1970s and was withdrawn from residential use by the 1990s. However, due to a long half-life, dieldrin persists in the environment

This work was funded by the Natural Sciences and Engineering Research Council of Canada [Grant RGPIN-2014-03687 to T.P.] and the Health and Life Sciences Seed Funding (to T.P.) and Canada Research Chair funding to C.J.M. L.S. was supported by the Beatrice Hunter Cancer Research Institute and the New Brunswick Health Research Foundation, and A.C. was supported by a Dalhousie Medicine New Brunswick Graduate Studentship.

https://doi.org/10.1124/jpet.116.239806.

S This article has supplemental material available at jpet.aspetjournals.org.
(Jorgenson, 2001) and has been associated with human diseases such as Alzheimer's disease (Singh et al., 2012, 2013), Parkinson's disease (Fleming et al., 1994; Weisskopf et al., 2010; Baltazar et al., 2014), and obesity (Cupul-Uicab et al., 2013). Short-term (Hung et al., 2015) and long-term (Sergeev and Carpenter, 2005; Kim et al., 2015) pesticide exposure are also associated with cardiovascular disease outcomes. Notably, prenatal exposure to pesticides disrupts cardiovascular development with significant relation to congenital heart disease (Gorini et al., 2014). Thus, studies are warranted to determine the mechanisms related to pesticide-induced cardiotoxicity in both the juvenile and adult heart.

Exposure to dieldrin and several other OCPs have been associated with cardiovascular disease. For instance, elevated circulating levels of OCPs are associated with peripheral artery disease (Min et al., 2011), insulin resistance (Lee et al., 2011), and cardiovascular disease development

ABBREVIATIONS: ACE, angiotensin-converting enzyme; Akt, protein kinase B; ANOVA, analysis of variance; AP-1, activator protein 1; cRNA, complementary RNA; hsc70, heat shock cognate $70 \mathrm{kDa}$; kcnh2a, potassium voltage-gated channel, subfamily $\mathrm{H}$ (eag-related), member 2a; kcnj11l, potassium inwardly rectifying channel, subfamily J, member 11, like; LAMP-2A, lysosomal-associated membrane protein 2 A; LC3, microtubuleassociated protein, light chain 3; MCOLN, mucolipin; mTOR, mechanistic target of rapamycin; OCP, organochlorine pesticide; phospho, phosphorylated; p70s6k, ribosomal s6 kinase; RIN, RNA integrity number; s6k, ribosomal S6 kinase 70 kDa; SNEA, subnetwork enrichment analysis; v-ATPase, vacuolar H+-ATPase; VF, ventricular fibrillation. 
(Ljunggren et al., 2014). Additionally, sublethal endosulfan exposure in rabbits results in myocardial apoptosis (Ozmen, 2013), whereas long-term lindane exposure in rats led to oxidative stress and loss of myofibril integrity (Ananya et al., 2005). At the molecular level, dieldrin exhibits toxicity by targeting mitochondria and the endoplasmic reticulum (Kitazawa et al., 2003). Dieldrin exposure also activates the protein kinase B (Akt)/mechanistic target of rapamycin (mTOR) pathway, a major regulator of cell growth and proliferation (Briz et al., 2011). The Akt/mTOR pathway also inhibits lysosomal autophagy (Jung et al., 2010). Autophagy, an evolutionarily conserved cellular mechanism for the lysosomal degradation of cytosolic components, including proteins and organelles, is known to respond to cell stress, including exposure to environmental pollution, and the failure of this response could result in proteotoxicity (Moore et al., 2006). Autophagy ensures cardiomyocyte survival by clearing toxic protein aggregates that accumulate in the cell during cardiac stress (Martinet et al., 2009). However, whether dieldrin exposure induces cardiac stress by altering autophagy is currently unknown.

We hypothesized that activation of the Akt/mTOR pathway by dieldrin suppresses lysosomal gene expression and autophagy signaling. To examine the cardiac effects of dieldrin, we used adult zebrafish (Danio rerio), a freshwater teleost that is a popular model for studying human diseases (Lieschke and Currie, 2007) and for screening and validating drugs and chemicals inducing cardiotoxicity (Milan et al., 2006; McGrath and Li, 2008; Liu et al., 2014). At the genetic level, approximately $70 \%$ of human genes have a zebrafish ortholog, and $82 \%$ of human genes with a known relation to morbidity have at least one zebrafish ortholog (Howe et al., 2013); thus, the zebrafish is a relevant model for human disease. Here, we show that dietary dieldrin exposure is sufficient to elevate mTOR signaling and its downstream targets, reducing transcripts related to autophagy and inducing aberrations in transcripts governing functional outcomes in the zebrafish heart.

\section{Materials and Methods}

\section{Experimental Design}

Six-month-old zebrafish (AB strain) were fed feed mixed with dieldrin at different concentrations for 21 days. Water parameters (mean \pm S.D.) during the experiment were as follows: temperature $=$ $25.5 \pm 1.5^{\circ} \mathrm{C}$, dissolved oxygen $=84.1 \pm 8.4 \%$, and $\mathrm{pH}=6.54 \pm 0.57$. Fish were housed with a photoperiod of 16 hours of light and 8 hours of dark. All procedures involving animals conformed to Canadian Council on Animal Care standards for fish handling and were approved by the University of New Brunswick, Saint John, animal care committee. To ensure that fish were exposed to dieldrin only through diet, $50 \%$ of the water in the tanks was changed every 3 days, and uneaten food and fish waste products were removed. Additionally, activated carbon was added to the tanks to prevent exposure to dieldrin from the water, which is effective (Bandala et al., 2006). Dieldrin was incorporated into the feed through dissolution in olive oil and then mixed with the diet, whereas the control diet was coated with olive oil only. The analytical chemistry analysis for feed has been published elsewhere (Cowie et al., 2017). Briefly, control feed contained no detectable dieldrin, whereas the mean dieldrin concentrations in feed were $0.03,0.15$, and $1.8 \mu \mathrm{g} / \mathrm{g}$ dry-weight feed for the low, medium, and high doses, respectively. The treatments were significantly different from the control group, and in this article the feed concentrations are used to describe treatments. Dieldrin was also measured in whole animals. Dieldrin in zebrafish that had been fed the control diet was at the detection limit, whereas the concentration in fish was $0.011 \mu \mathrm{g}$ dieldrin/g dry-weight in low-dose, $0.058 \mu \mathrm{g}$ dieldrin/g dry-weight in medium-dose, and $0.47 \mu \mathrm{g}$ dieldrin/g dryweight in high-dose treatments. The mean wet-weight concentrations of dieldrin in whole zebrafish that were fed the $0.03,0.15$, and $1.8 \mu \mathrm{g} / \mathrm{g}$ doses were $3.50,17.6$, and $148 \mathrm{ng} / \mathrm{g}$ wet-weight, respectively. Both the medium- and high-dose treatment groups were significantly different from control fish after 21 days of treatment, whereas the low-dose treatment group was not different from controls. After 21 days, the fish were euthanized using a sodium bicarbonate buffered solution of $250 \mathrm{mg} / \mathrm{l}$ Tricaine (Sigma-Aldrich, St. Louis, MO) and subsequent severing of the spinal cord. Hearts were dissected from the animals, flash frozen in dry ice, and stored at $-80^{\circ} \mathrm{C}$ until processed.

\section{Expression Profiling in Dieldrin-Treated Zebrafish Hearts}

RNA Extraction from Whole Zebrafish Hearts. Female zebrafish hearts were pooled into 15 tubes. Final sample sizes for microarray analysis were as follows: control group $(n=5)$, fish fed the 0.15 $\mu \mathrm{g} / \mathrm{g}$ dose diet $(n=6)$, and fish fed the $1.8 \mu \mathrm{g} / \mathrm{g}$ dose diet $(n=4)$. Consideration was made for keeping the pooled masses even across samples. RNA was extracted using the RNeasy Mini Kit (Qiagen, Toronto, ON, Canada). The concentration of RNA was measured using the BioTek Synergy H4 and Take3 plate by assessing absorbance at $260 \mathrm{~nm}$. RNA quality was evaluated using the 2100 Bioanalyzer (catalog \#G2940CA; Agilent, Santa Clara, CA), which produces an RNA integrity number (RIN). RINs greater than 7.5 were deemed acceptable for microarray analysis (Kiewe et al., 2009). The mean ( \pm S.D.) RIN value was $9.75 \pm 0.21$ for female heart pools, and RIN values in samples ranged in from 9.4 to 10 .

Sample Labeling. RNA samples were labeled with Cyanine-3 using the Low Input Quick Amp Labeling Kit for one-color microarray (catalog \#5190-2305; Agilent) according to manufacturer instructions, starting with $45 \mathrm{ng}$ of RNA input. Quantification of the labeled cRNA was performed using the Nanodrop 2000 Spectrophotometer (Thermo Scientific, Buckinghamshire, UK). Cyanine 3 dye concentration (in picomoles per microliter), and cRNA concentration were measured for each sample. The total yield of cRNA (in micrograms) and the specific activity of $\mathrm{Cy} 3$ (concentration of $\mathrm{Cy} 3 /$ concentration of cRNA) were calculated to determine whether labeling and amplification were successful. Microarray analysis with Agilent $4 \times 44,000$ microarrays proceeded if the total yield of cRNA was greater than $1.65 \mu \mathrm{g}$ and the specific activity of $\mathrm{Cy} 3$ was greater than $6 \mathrm{pmol} / \mu \mathrm{g}$ cRNA, criteria that were met by all samples.

Microarray Hybridization and Analysis. Microarray hybridization followed the Agilent One-Color Microarray-Based Gene Expression Analysis protocol for Agilent $4 \times 44,000$ zebrafish version 3 microarray slides. After hybridization, slides were scanned with the Agilent Microarray Scanner at a resolution of $5 \mu \mathrm{m}$. Raw expression data along with tif images were extracted by Agilent Feature Extraction Software (version 10.7.3.1). All microarray data reported in this study follow established guidelines "Minimum Information About a Microarray Experiment (MIAME)" and are located in the Gene Expression Omnibus (\#GSE87294; Gene Ontology Consortium 2000). The arrays were quality control checked using a distribution analysis that plots the intensity distributions of each microarray slide to ensure that these distributions are relatively equal. One microarray in the control group showed localized intensity outliers in the center of the array, and these features were manually removed from the analysis. Box plots of normalized intensity also showed that the intensity distribution was not different across slides. The microarray data were analyzed using JMP Genomics (version 6; SAS, Cary, NC) to identify differentially expressed genes using a one-way analysis of variance (ANOVA) followed by a determination of false discovery rate (5\%). Data were first normalized using Quantile normalization and probes falling 

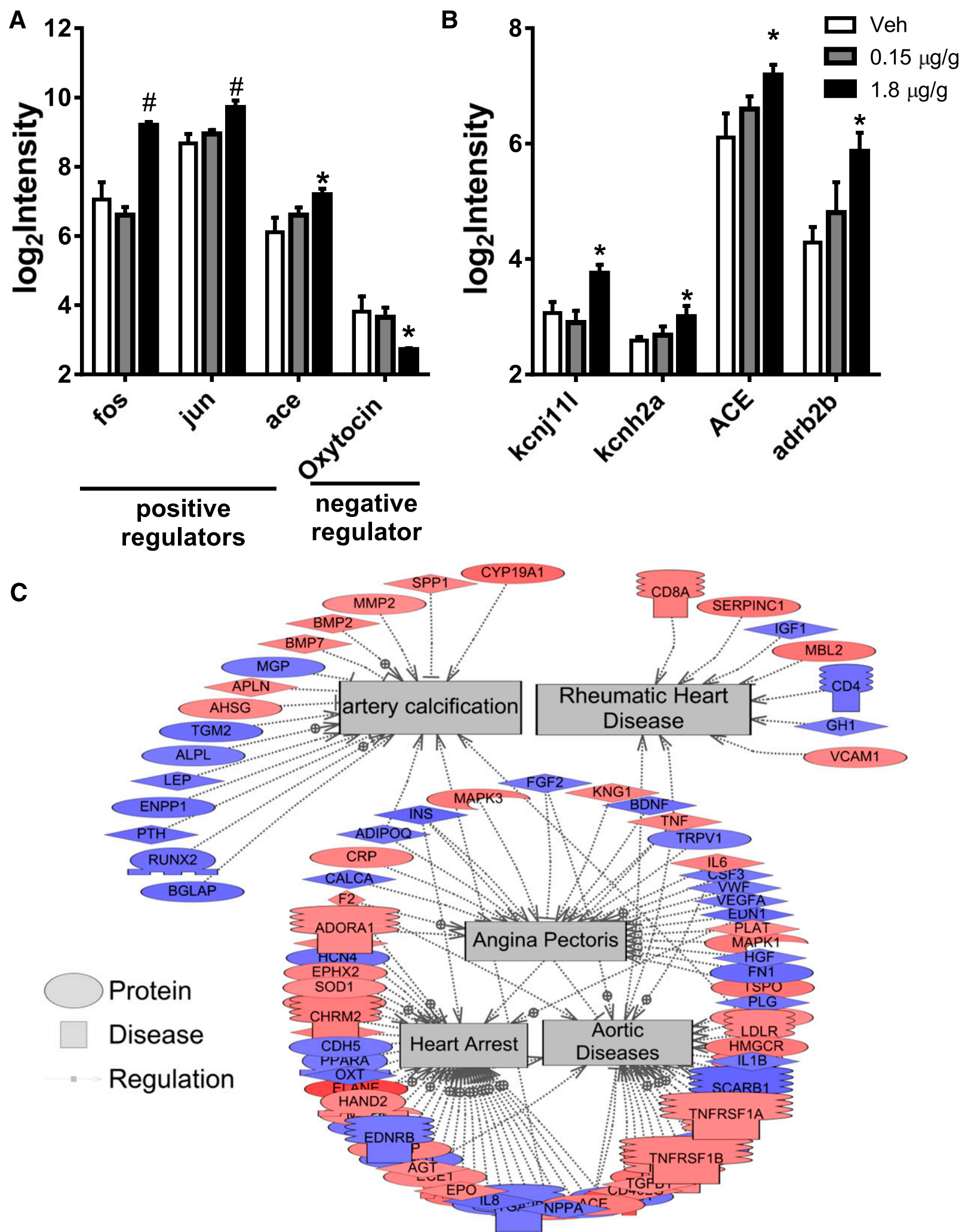

Fig. 1. Mean $\log 2$ intensity plus S.E.M. of genes related to diseases as identified by gene expression microarrays and SNEA in female zebrafish hearts treated with a $1.8 \mu \mathrm{g} / \mathrm{g}$ (black bars) or $0.15 \mu \mathrm{g} / \mathrm{g}$ (gray bars) dose of dieldrin or vehicle control (Veh; white bars) for 21 days, with $* P<0.05$, \#P $<0.01$ compared with the control group. (A) Gene regulating cardiac arrest separated into positive and negative regulators. (B) Genes positively regulating VF (C) Expression changes of genes related to cardiovascular diseases, which were differentially expressed by the $1.8 \mu \mathrm{g} / \mathrm{g}$ dose of dieldrin in the adult zebrafish heart. Red signifies upregulation, blue signifies downregulation, and definitions of abbreviations can be found in Supplemental Appendix 3 .

below the detection limit of the microarrays (2.5) were assigned an intensity value of 2.5 . Bioinformatic analysis [subnetwork enrichment analysis (SNEA) and expression targets] was performed in
Pathway Studio 9.0 [Ariadne, Rockville, MD (now Elsevier)] using the ResNet 9.0, a mammalian database curated by Ariadne (Nikitin et al., 2003). 
TABLE 1

Diseases identified with SNEA from transcriptomics of female zebrafish

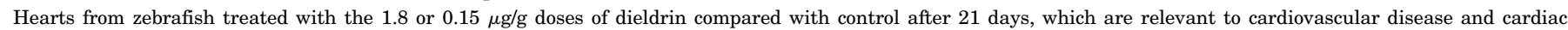
physiology. Values are reported as the number of genes in the network and the number of measured genes on the zebrafish platform, unless otherwise indicated.

\begin{tabular}{|c|c|c|c|c|}
\hline Name & Total Neighbors & Measured Neighbors & Median Fold Change of Network & $P$ value \\
\hline & & $n$ & & \\
\hline \multicolumn{5}{|c|}{ Cardiovascular diseases regulated by the high dose } \\
\hline Arterial stiffness & 32 & 23 & 1.04 & 0.003 \\
\hline Heart arrest & 45 & 36 & 1.16 & 0.009 \\
\hline Aortic diseases & 44 & 26 & 1.25 & 0.013 \\
\hline Rheumatic heart disease & 11 & 9 & 1.38 & 0.013 \\
\hline Artery rhythm & 10 & 7 & -1.17 & 0.017 \\
\hline Angina pectoris & 28 & 22 & -1.11 & 0.019 \\
\hline Weight gain & 179 & 136 & 1.02 & 0.039 \\
\hline Cardiogenic shock & 7 & 5 & -1.31 & 0.042 \\
\hline Diabetes mellitus & 20 & 12 & 1.11 & 0.043 \\
\hline \multicolumn{5}{|c|}{ Cardiovascular diseases regulated by the medium dose } \\
\hline Ventricular Fibrillation & 43 & 30 & 1.17 & 0.006 \\
\hline Venous thromboembolism & 16 & 15 & 1.02 & 0.034 \\
\hline Carotid artery diseases & 18 & 14 & 1.04 & 0.034 \\
\hline
\end{tabular}

\section{Gel Electrophoresis and Immunoblotting}

Frozen whole zebrafish hearts ( 80 in total) were pooled into tubes on the basis of the tank they originated from while keeping sexes separate [i.e., two separate lysates (male or female) were generated from one tank (five fish), for a total of four female and four male biologic replicates per treatment]. The pooled hearts were homogenized in $60 \mu \mathrm{l}$ of ice-cold lysis buffer, as previously described (Bartlett et al., 2016). The homogenates were allowed to settle on ice before being centrifuged for 30 minutes at $1200 \mathrm{~g}$. The supernatant was aspirated from the pellet and stored in $1.5-\mathrm{ml}$ centrifuge tubes at $-80^{\circ} \mathrm{C}$.

Total protein concentration in pooled heart samples was estimated using the Pierce BCA Protein Assay Kit (catalog \#23225; ThermoFisher Scientific, Waltham, MA). Protein samples were size fractionated with SDS-PAGE using Any kD Criterion Pre-Cast Gradient Gels (catalog \#567-8125; Bio-Rad, Hercules, CA) before being electroblotted onto a nitrocellulose membrane. The uniformity and effectiveness of transfer were assessed by a Pierce reversible protein stain kit for nitrocellulose membranes (ThermoFisher Scientific) and were imaged with the ChemiDoc MP Imaging System (Bio-Rad) before the stain was washed away. Blotted proteins were characterized using the following primary antibodies: phospho-mTOR, Ser2448 (catalog \#2971; Cell Signaling Technology, Danvers, MA); phospho-Akt, Ser473 (catalog \#9271; Cell Signaling Technology); phospho-s6k, Thr389 (catalog \#9205; Cell Signaling Technology); s6k (catalog \#2708; Cell Signaling Technology); lysosomal-associated membrane protein 2A (LAMP-2A) (catalog \#AB18528; Abcam, Cambridge, UK); heat shock cognate $70 \mathrm{kDa}$ (hsc70) (catalog \#AB2788; Abcam); and Cathepsin D (catalog \#SC-6486; Santa Cruz Biotechnology, Dallas, TX). Immunoblots were developed with the Pierce SuperSignal West Dura Substrate (catalog \#34075; ThermoFisher Scientific) and were imaged with the Bio-Rad ChemiDoc MP Imaging System. Normalized signal intensity of quantified immunoblots was analyzed using GraphPad Prism 6 (GraphPad, La Jolla, CA). A two-way ANOVA was conducted to test the effect of sex on dieldrin treatment; however, the factor was not significant and there was no interaction. One-way ANOVA was used to determine whether the groups (comprised of two male samples and two female samples) were significantly different and, if so, whether a Dunnett's post hoc test or unpaired two-tailed $t$ tests were used to compare individual groups.

\section{TABLE 2}

Cell processes identified with subnetwork enrichment analysis from transcriptomics of female zebrafish hearts treated with the 1.8 or $0.15 \mu \mathrm{g} / \mathrm{g}$ doses of dieldrin compared with control after 21 days

\begin{tabular}{|c|c|c|c|c|c|c|}
\hline \multirow{2}{*}{ Name } & \multirow{2}{*}{ Total Neighbors } & \multirow{2}{*}{ Measured Neighbors } & \multicolumn{2}{|c|}{ Median Fold Change } & \multicolumn{2}{|c|}{$P$ Value } \\
\hline & & & $1.8 \mu \mathrm{g} / \mathrm{g}$ & $0.15 \mu \mathrm{g} / \mathrm{g}$ & $1.8 \mu \mathrm{g} / \mathrm{g}$ & $0.15 \mu \mathrm{g} / \mathrm{g}$ \\
\hline & & $n$ & & & & \\
\hline \multicolumn{7}{|c|}{ Cell processes regulated by both 1.8 and $0.15 \mu \mathrm{g} / \mathrm{g}$ doses } \\
\hline Endocrine changes & 15 & 14 & 1.3 & -1.15 & 0.0059 & 0.0048 \\
\hline Muscle metabolism & 37 & 36 & -1.19 & -1.15 & 0.0079 & 0.031 \\
\hline Lipid modification & 12 & 9 & -1.2 & -1.22 & 0.0085 & 0.0067 \\
\hline Lipid export & 93 & 67 & -1.07 & -1.07 & 0.021 & 0.0012 \\
\hline Lipoprotein metabolism & 103 & 67 & 1.01 & -1.03 & 0.025 & 0.042 \\
\hline \multicolumn{7}{|c|}{ Cell processes regulated by $1.8 \mu \mathrm{g}$ dieldrin/g food } \\
\hline Heart muscle growth & 5 & 5 & 1.5 & & 0.0039 & \\
\hline Muscle development & 178 & 148 & 1.01 & & 0.0065 & \\
\hline Myocyte function & 93 & 74 & -1.01 & & 0.0071 & \\
\hline Fatty acids import & 84 & 64 & -1.07 & & 0.014 & \\
\hline Hormone metabolism & 30 & 18 & 1.2 & & 0.018 & \\
\hline Lysosomal lumen acidification & 38 & 28 & 1.08 & & 0.027 & \\
\hline Cell dedifferentiation & 47 & 31 & 1.10 & & 0.03 & \\
\hline Inflammatory response & 1087 & 703 & 1.001 & & 0.041 & \\
\hline \multicolumn{7}{|c|}{ Cell processes regulated by $0.15 \mu \mathrm{g}$ dieldrin $/ \mathrm{g}$ food } \\
\hline Fatty acid metabolism & 156 & 118 & & 1.06 & & 0.0075 \\
\hline Vasculature development & 20 & 15 & & 1.03 & & 0.016 \\
\hline Lipid metabolism & 470 & 345 & & 1.02 & & 0.034 \\
\hline Artery remodeling & 80 & 61 & & -1.09 & & 0.043 \\
\hline
\end{tabular}




\section{Results}

Transcriptomics Analysis of the Zebrafish Heart after Dieldrin Exposure. Analysis of gene expression in response to dieldrin treatment resulted in 627 differentially expressed transcripts by either the measured 1.8 or $0.15 \mu \mathrm{g} / \mathrm{g}$ dose of dieldrin treatment $(P<0.01)$. Furthermore, between the $1.8 \mu \mathrm{g} / \mathrm{g}$ dose and the $0.15 \mu \mathrm{g} / \mathrm{g}$ dose of dieldrin, there were 47 transcripts differentially expressed $(P<0.01)$. Transcripts significantly regulated by both doses include myosin, light chain $9 b$, regulatory, which is involved in muscle contraction, and cyclin-dependent kinase inhibitor $1 b$, which is involved in cell cycle arrest. Furthermore, two transcripts that are associated with endosome regulation, mucolipin (mcoln)-3 and map1lc3a, were downregulated in the heart after both treatment doses of dieldrin. All microarray gene expression data can be found in Supplemental Appendix 1. After applying a stringent false discovery rate of 5\%, one transcript in the $1.8 \mu \mathrm{g} / \mathrm{g}$ dieldrin treatment group, sphingosine-1-phosphate receptor $5 b$, was significantly different compared with the control group. Pathway analysis, however, was conducted on all of the transcriptomic data as per our established methods (Cowie et al., 2017).

SNEA revealed that gene networks associated with 225 diseases were altered in the heart by either the 1.8 or $0.15 \mu \mathrm{g} / \mathrm{g}$ dose of dieldrin, and gene networks associated with 39 diseases were altered in zebrafish heart using both doses. All SNEAs for diseases can be found in Supplemental Appendix 2. Several of the disease networks identified by SNEA pertained to cardiovascular pathophysiology, including artery calcification, rheumatic heart disease, angina pectoris, and cardiac arrest (Fig. 1C; Table 1). Specific genes related to cardiac arrest included the complex of fos (FBJ murine osteosarcoma viral oncogene homolog; fold change $=4.42, P=0.001$ ) and jun (fold change $=2.04, P=0.005$ ), two proteins that dimerize to form a transcription factor regulating cell proliferation. Furthermore, angiotensin-converting enzyme (ace) expression, a positive regulator of blood pressure, was increased by $>2$-fold (fold change $=2.11, P=0.04$ ), while oxytocin, a negative regulator of blood pressure, was decreased $>2$-fold (fold change $=-2.14, P=0.048$ ) (Fig. 1A). Transcripts related to ventricular fibrillation (VF) were also identified as being altered by dieldrin. These genes included ace, as well as catecholamine receptor adrenoceptor $\beta 2 a b$ (fold change $=2.99, P=0.021$ ). Two potassium channels specific to myocytes, which regulate membrane potential, were also upregulated with dieldrin, including potassium inwardly rectifying channel, subfamily J, member 11, like (kcnj11l) was increased (fold change $=1.61, P=0.03$ ) along with potassium voltage-gated channel, subfamily $H$ (eagrelated), member $2 a, k c n h 2 a($ fold change $=1.33, P=0.049)$ (Fig. 1B).

Dieldrin Treatment Alters Inflammatory and Lysosomal Processes. Analysis of microarray results by SNEA revealed that 196 cell processes were regulated by the $1.8 \mu \mathrm{g} / \mathrm{g}$ dose of dieldrin, whereas 129 cell processes were regulated by the $0.15 \mu \mathrm{g} / \mathrm{g}$ dose in zebrafish hearts at $P<0.05$. SNEA showed that 56 cell processes were regulated by both the 0.15 and $1.8 \mu \mathrm{g} / \mathrm{g}$ doses. All SNEAs for cell processes can be found in Supplemental Appendix 2. Cell processes that we determined to be related to cardiovascular metabolism (Table 2) were further investigated to identify their component genes.
Processes regulating inflammatory stress were significantly upregulated by dieldrin $(P=0.041)$. Specific genes that were altered by treatment with the $1.8 \mu \mathrm{g} / \mathrm{g}$ dose of dieldrin include the following positive mediators of inflammation: interleukin 6 (fold change $=1.46, P=0.034$ ) and tumor necrosis factor$\alpha$-induced protein 2 (fold change $=1.24, P=0.020)($ Fig. $2 \mathrm{~A}$ ). Negative regulators of inflammation, such as nuclear factor$\kappa B$-repressing factor (fold change $=-1.15, P=0.047$ ) and nuclear factor $-\kappa B$ inhibitor interacting Ras-like (fold change $=$ $-1.2, P=0.042$ ) (Fig. 2A), were downregulated in the heart by the $1.8 \mu \mathrm{g} / \mathrm{g}$ dose of dieldrin. Enrichment analysis also identified several processes related to lipid metabolism that were significantly altered in the heart by the $1.8 \mu \mathrm{g} / \mathrm{g}$ dose of dieldrin (Fig. 3). Other cell processes identified as being differentially upregulated in the heart by the $1.8 \mu \mathrm{g} / \mathrm{g}$ dose of dieldrin include heart muscle growth and muscle development, along with a downregulation of processes involved in myocyte function (Table 2), which, taken together with the diseases identified as being differential regulated, indicate that dieldrin exposure renders zebrafish cardiomyocytes susceptible to injury.

SNEA also identified gene networks related to protein degradation, specifically those pertaining to lysosomal lumen acidification and autophagy. Transcripts that play a role in
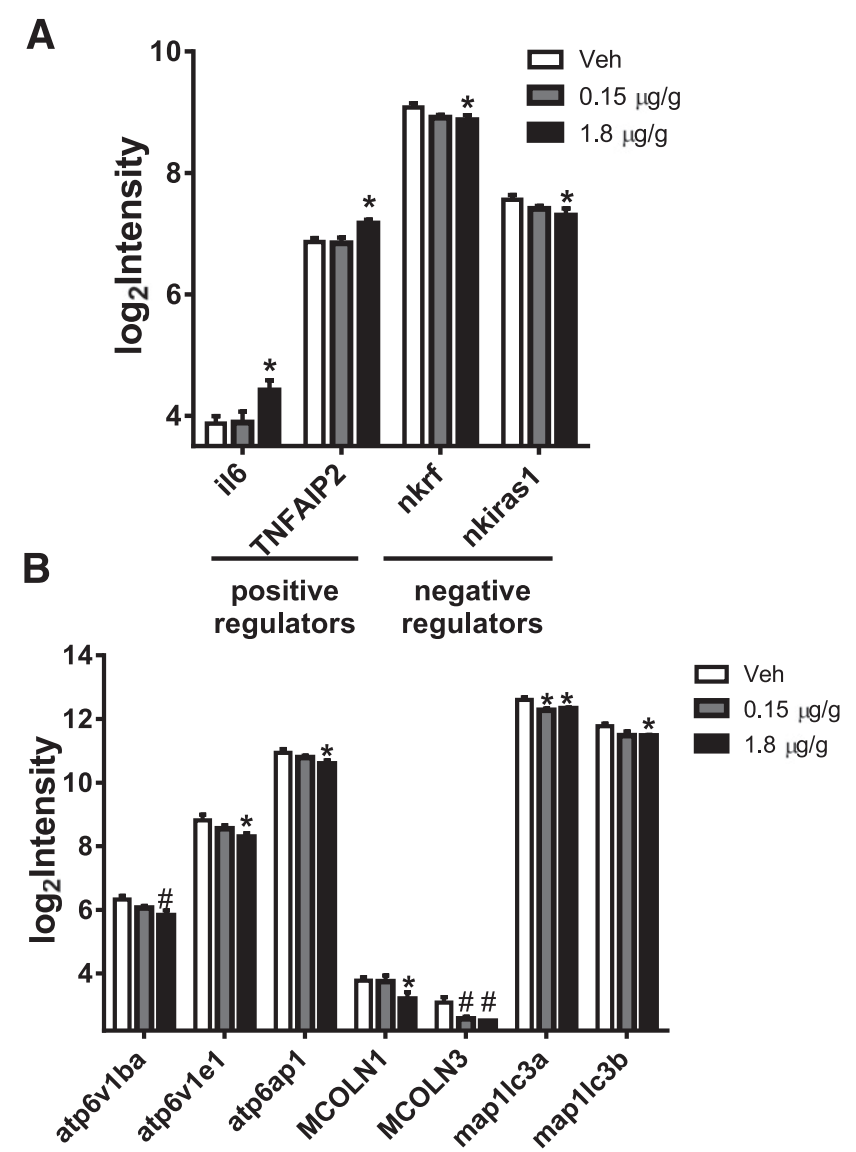

Fig. 2. Mean $\log 2$ intensity plus S.E.M. for genes related to cell processes as identified by gene expression microarrays and SNEA in female zebrafish hearts treated with a $1.8 \mu \mathrm{g} / \mathrm{g}$ (black bars) or $0.15 \mu \mathrm{g} / \mathrm{g}$ (gray bars) dose of dieldrin or vehicle control (Veh; white bars) for 21 days, with $* P<0.05, \# P<0.01$ compared with the control group. (A) Genes regulating inflammation separated into positive and negative regulators. (B) Gene regulating lysosomal function and acidification. 


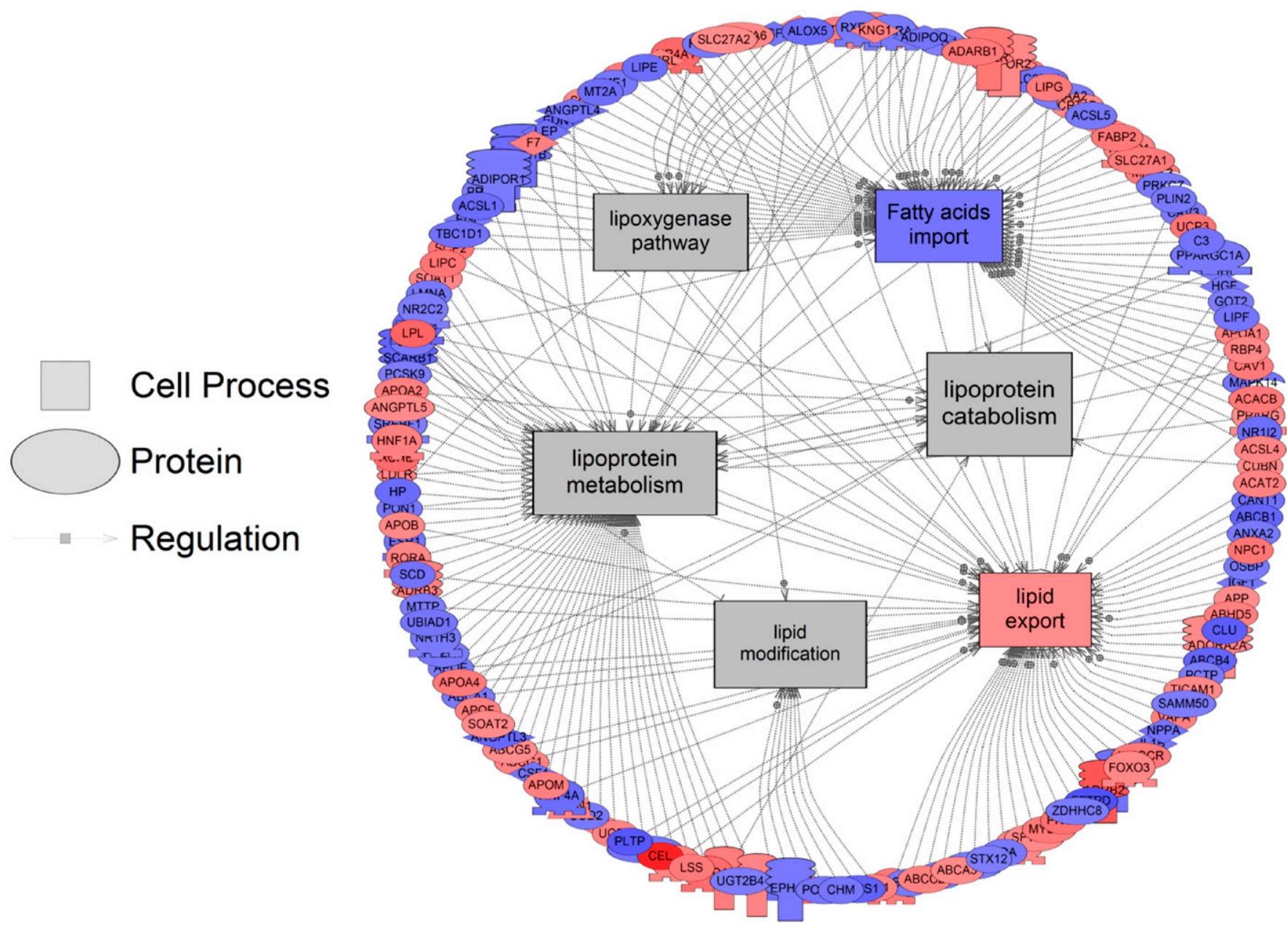

Fig. 3. Expression changes of genes related to lipid metabolism, which were differentially expressed by the $1.8 \mu \mathrm{g} / \mathrm{g}$ dose of dieldrin in the adult zebrafish heart. Red signifies upregulation, blue signifies downregulation, and definitions of abbreviations can be found in Supplemental Appendix 3 .

these processes are differentially regulated by dieldrin (Table 2). Significantly altered transcripts related to lysosomal lumen acidification include the lysosomal $\mathrm{H}+$ transport proteins, atp6v1ba, atp6v1e1, and atp6ap1, which were all significantly $(P<0.05)$ downregulated in the heart by the 1.8 $\mu \mathrm{g} / \mathrm{g}$ dose of dieldrin. Furthermore, two membrane proteins required for lysosomal trafficking and acidification, mcoln1 and $m c o l n 3$, are downregulated in the heart after the $1.8 \mu \mathrm{g} / \mathrm{g}$ dose of dieldrin (fold change $=-1.49$ and $-1.50, P=0.039$ and 0.004 , respectively), whereas mcoln 3 is also downregulated in the heart by the $0.15 \mu \mathrm{g} / \mathrm{g}$ treatment (fold change $=-1.42, P=$ $0.005)$. We observed that the expression of genes involved in lysosomal autophagy were significantly downregulated in the heart in response to dieldrin, and these included microtubuleassociated protein, light chain $3 A$ and $B(L C 3 A / B)$. Lc $3 a$ was decreased in the heart by both doses of dieldrin $(1.8 \mu \mathrm{g} / \mathrm{g}$ dose: fold change $=-1.21, P=0.009 ; 0.15 \mu \mathrm{g} / \mathrm{g}$ dose: fold change $=$ $-1.26, P=0.001$ ), whereas $l c 3 b$ was decreased in the fish fed $0.15 \mu \mathrm{g} / \mathrm{g}$ dieldrin in the feed (fold change $=-1.23, P=0.047$ ) (Fig. 2B).

Dieldrin Treatment Increases mTOR Signaling. Given that genes critical for lysosomal function were identified by microarray analysis as being suppressed at the transcript level by dieldrin, we more closely examined the pathway that regulates the lysosome $(\mathrm{Akt} / \mathrm{mTOR})$ at the protein level. Immunoblotting for the mTOR and its upstream and downstream targets revealed hyperphosphorylation and activation of the pathway in dieldrin-treated zebrafish hearts (Fig. 4). Akt, an upstream activator of mTOR, shows a significant $(F(3,12)=5.502, P=0.013)$ increase in phosphorylation at serine $473 \mathrm{in}$ fish fed both the $1.8 \mu \mathrm{g} / \mathrm{g}(P<0.05)$ and $0.15 \mu \mathrm{g} / \mathrm{g}(P<0.01)$ doses compared with controls (Fig. 4B). Likewise, mTOR phosphorylation was significantly $(F(3,12)=$ $3.517, P=0.049)$ increased in hearts from the $1.8 \mu \mathrm{g} / \mathrm{g}$ group $(P<0.05)$ compared with the control group (Fig. 4C). The downstream target of mTOR, ribosomal s6 kinase (p70s6k) is also significantly $(F(3,12)=4.709, P=0.021)$ hyperphosphorylated in zebrafish from the $1.8 \mu \mathrm{g} / \mathrm{g}$ treatment group $(P<0.01)$ compared with the control group (Fig. 4D). These results correspond to our microarray findings, which indicated that expression targets of p70S6K and p90S6K (downstream targets of mTOR), negative regulators of autophagy, are upregulated in fish in the $1.8 \mu \mathrm{g} / \mathrm{g}$ treatment group (Fig. 5). All expression target data can be found in Supplemental Appendix 2.

To test our hypothesis that dieldrin alters lysosomal function, which was suggested by the microarray analysis, we immunoblotted for autophagy-related targets (Fig. 6). We determined that the expression of cathepsin $\mathrm{D}$, a lysosomal protease, has significantly reduced protein expression 


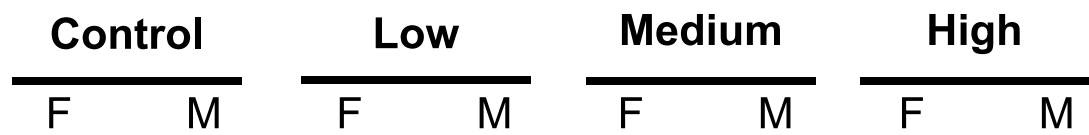

P-AKT Ser 473

P-mTOR Ser 2448

Protein Stain

\section{P-P70S6K Thr ${ }^{389}$}

P70S6K

Protein Stain

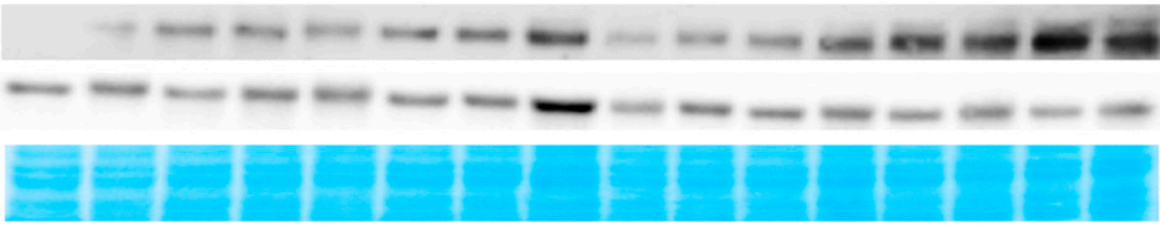

B phospho-AKT (Ser473)

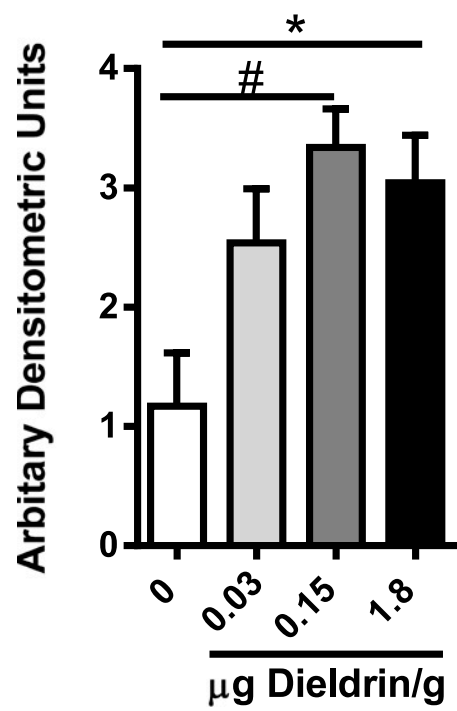

C phospho-mTOR (Ser2448)

D

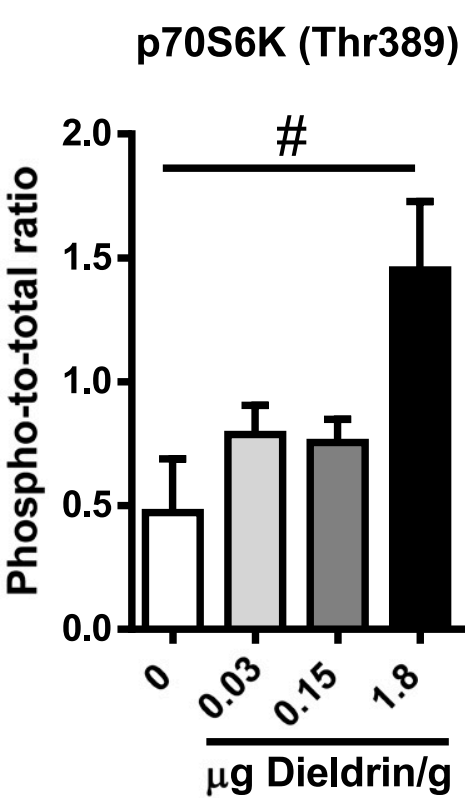

Fig. 4. Immunoblot analysis of Akt/mTOR pathway protein phosphorylation in pooled zebrafish hearts treated with three different concentrations of dieldrin or control for 21 days. $* P<0.05$; $\# P<0.01$. (A) Immunoblot images of protein targets in the four different concentrations, along with protein stain loading control separated by treatment group and sex. F, female; M, male. (B) Phospho-Akt (Ser473) immunoblot quantification after correction to protein stain. (C) Phospho-mTOR (Ser2448) immunoblot quantification after correction to protein stain. (D) Quantification of phospho-p70s6k immunoblot by correcting the quantity to unphosphorylated p70s6k, mean densitometric units plus S.E.M. $n=4$ per treatment group. P, phospho.

$(F(3,12)=3.662, P=0.044)$ in zebrafish from both the 1.8 and $0.15 \mu \mathrm{g} / \mathrm{g}$ dieldrin treatment groups compared with the control group $(P<0.05, t$ test) (Fig. 6B). Immunoblotting for LAMP-2A and hsc70 showed that levels were unchanged in fish from the dieldrin-treated groups compared with the control group (Fig. 6, C and D). However, the gene expression analysis identified macroautophagy transcripts to be differentially regulated, whereas we were only able to examine the expression of chaperone-mediated autophagy proteins.

\section{Discussion}

The overall goal of this study was to determine whether dieldrin at a low, environmentally relevant dose results in cardiac injury. To achieve this, we examined the effect of a 3-week dieldrin (model legacy OCP) exposure in the hearts of zebrafish. The total body burden of dieldrin achieved by the 3 -week feeding is comparable to what is observed in some wild fish. Dieldrin total body burden in largemouth bass after 4 months of exposure at a contaminated site was roughly $500 \mathrm{ng} / \mathrm{g}$ wet-weight (Martyniuk et al., 2010b) compared with our study in which we saw a total body burden of $\sim 150 \mathrm{ng} / \mathrm{g}$ wet-weight after 3 weeks of eating feed containing a $1.8 \mu \mathrm{g} / \mathrm{g}$ dose of dieldrin. It is more difficult to compare the concentrations achieved in our study to human health as most human data are based upon serum, breast milk, and individual tissue concentrations (Brock et al., 1998; Jorgenson, 2001; Fujii et al., 2012), whereas our study measured the whole-body burden exclusively. Another area of caution in this study is the sex-specific effect of dieldrin. Our gene expression analysis 


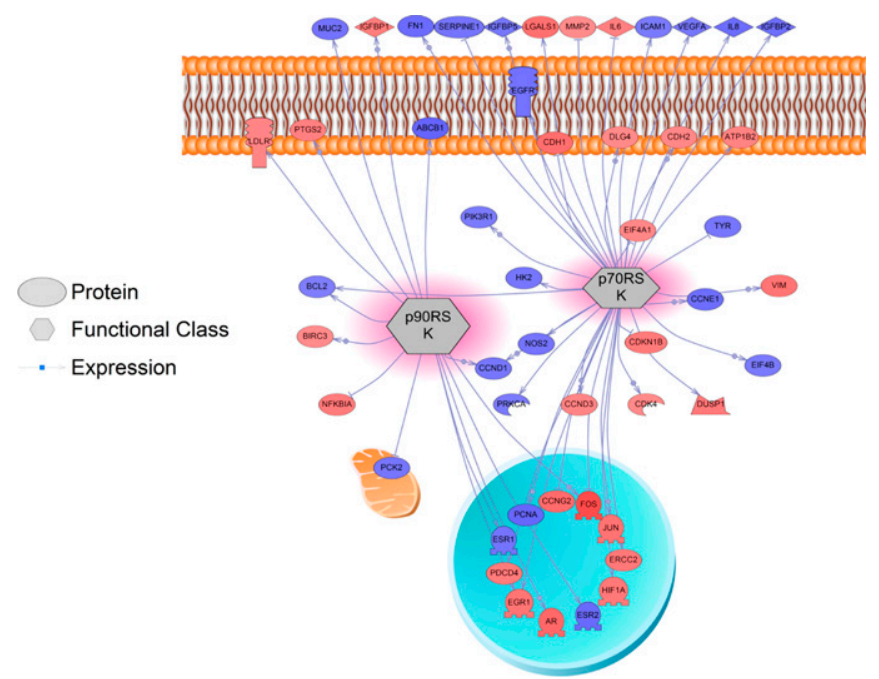

Fig. 5. Expression targets of p90RSK (ribosomal S6 kinase, $90 \mathrm{kDa}$ ) and p70RSK (ribosomal S6 kinase, $70 \mathrm{kDa}$ ), which were differentially expressed by the $1.8 \mu \mathrm{g} / \mathrm{g}$ dose of dieldrin in the adult zebrafish heart. Red signifies upregulation, blue signifies downregulation, and definitions of abbreviations can be found in Supplemental Appendix 3.

was performed on hearts from adult female zebrafish, whereas the Western blot analysis included both male and female fish. Given that previous studies have identified a sexually dimorphic response to dieldrin (Martyniuk et al., 2013), future experiments should investigate whether the same transcriptome networks are altered in male fish.

This is the first study to reveal that gene networks associated with cardiovascular disease, along with several cellular processes related to the development of cardiac injury, are altered by 3 weeks of dieldrin treatment in adult zebrafish. Together, these data suggest that dieldrin causes cardiomyocyte injury, which is due to altered status of growth, metabolic dysregulation, and lysosomal stress.

Our first aim was to characterize the global gene expression response in female zebrafish hearts to that after 21 days of dieldrin treatment and to use bioinformatics approaches to relate the gene expression data to cell processes and diseases. This is one of the few reports on the effect of dieldrin in the vertebrate heart. Others have reported that aldrin, dieldrin, and endrin all inhibit cardiac sarcoplasmic reticulum calcium pump activity in a dose-dependent manner (Mehrotra et al., 1989). Interestingly, our data also indicate that target genes regulating ion pump function are downregulated by dieldrin treatment. In the current study, we demonstrate via functional enrichment analysis of microarray data that alterations in gene networks regulating the susceptibility to cardiac arrest, VF, and arteriole stiffness are associated with dieldrin exposure.

Functional enrichment analysis identified several genes that are related to VF lethal form of cardiac arrhythmia. Notably kcnj11l, also known as Kir6.2, part of a family of potassium-ATP channels, was upregulated by dieldrin (Medeiros-Domingo et al., 2010). Interestingly, dieldrin also increased the expression of the voltage-gated potassium channel $k c n h 2$. Gain-of-function mutations for KCNH2 result in arrhythmias such as arterial fibrillation and short- or long-QT syndromes (Hong et al., 2005). Carriers of KCNH2 gain-of-function mutations have an increased chance of ventricular arrhythmias resulting in sudden cardiac death (Brugada et al., 2004). Furthermore, others found that downregulation of Kir6.2 resulted in the protection of ischemiainduced VF (Tsuburaya et al., 2011). We observe that the $1.8 \mu \mathrm{g} / \mathrm{g}$ dose of dieldrin also increases genes in networks related to cellular dedifferentiation in addition to muscle development and growth, which are processes that are characteristic of zebrafish heart regeneration in response to injury (Jopling et al., 2010).

Preclinical evidence from mammalian cardiovascular research indicates that vasculomuscular stiffness and injury in the heart is an outcome of excess inflammation. In our study, transcripts related to inflammatory response were shown to be modulated in the zebrafish heart after 21 days of treatment with dieldrin. Previous reports have suggested that shortterm dieldrin injection or long-term exposure activates a transcriptional inflammatory response in the largemouth bass hypothalamus (Martyniuk et al., 2010a,b). Likewise, dieldrin was able to induce proinflammatory cytokine production in human neutrophils along with the activation of other neutrophil functions (Pelletier et al., 2001). Our microarray results indicate that interleukin 6 is upregulated in response to dieldrin treatment. Notably, our data also show that fos and jun, which combine to form activator protein 1 (AP-1), which induces muscle differentiation and proliferation (Shaulian and Karin, 2002; Moore-Carrasco et al., 2006), are both significantly upregulated in response to dieldrin. AP-1 overexpression is associated with cardiac hypertrophy (Herzig et al., 1997) and chronic heart failure (Frantz et al., 2003). Interestingly, ace is upregulated by dieldrin in our results, and angiotensin II both activates and induces the expression of AP-1 (Herzig et al., 1997; van Wamel et al., 2001). ACE was also identified as a gene related to the development of arterial stiffness in zebrafish treated with the high dose of dieldrin. An inflammatory response initiated by dieldrin treatment may be another mechanism of AP-1 activation, considering that proinflammatory cytokines induce the expression of both FOS and JUN (Karin et al., 1997).

Downstream cellular effects of dieldrin involve changes in the mitochondrial and endoplasmic reticulum. Prolonged oxidative stress from dieldrin exposure may result in mitochondrial dysfunction and the release of cytochrome $\mathrm{C}$ into the cytosol, leading to apoptosis (Kitazawa et al., 2003). Likewise, treatment with endosulfan, which is in the same chemical class as dieldrin, results in increased caspase-3 expression in the heart (Ozmen, 2013). In another proposed mechanism, dieldrin causes endoplasmic reticulum stress, which leads to decreases in endoplasmic reticulum-associated protein degradation, resulting in proteotoxicity and cell death (Sun et al., 2005). We examined whether the disruption of lysosomal function and autophagy is a plausible outcome of dieldrin exposure in zebrafish hearts. Functional enrichment analysis identified an alteration in gene transcripts that regulate lysosomal lumen acidification wherein examinations of individual genes responsible for lysosomal acidification are downregulated in the heart. Our search identified that three lysosomal vacuolar H+-ATPases (v-ATPases) have significantly reduced expression in fish fed the high dieldrin dose. Lysosomal v-ATPase uses energy from ATP hydrolysis to pump protons into the lysosomal lumen, thereby regulating the lysosomal acidity (Forgac, 2007). Bafilomycin, a selective v-ATPase inhibitor, inhibits lysosomal acidification and 

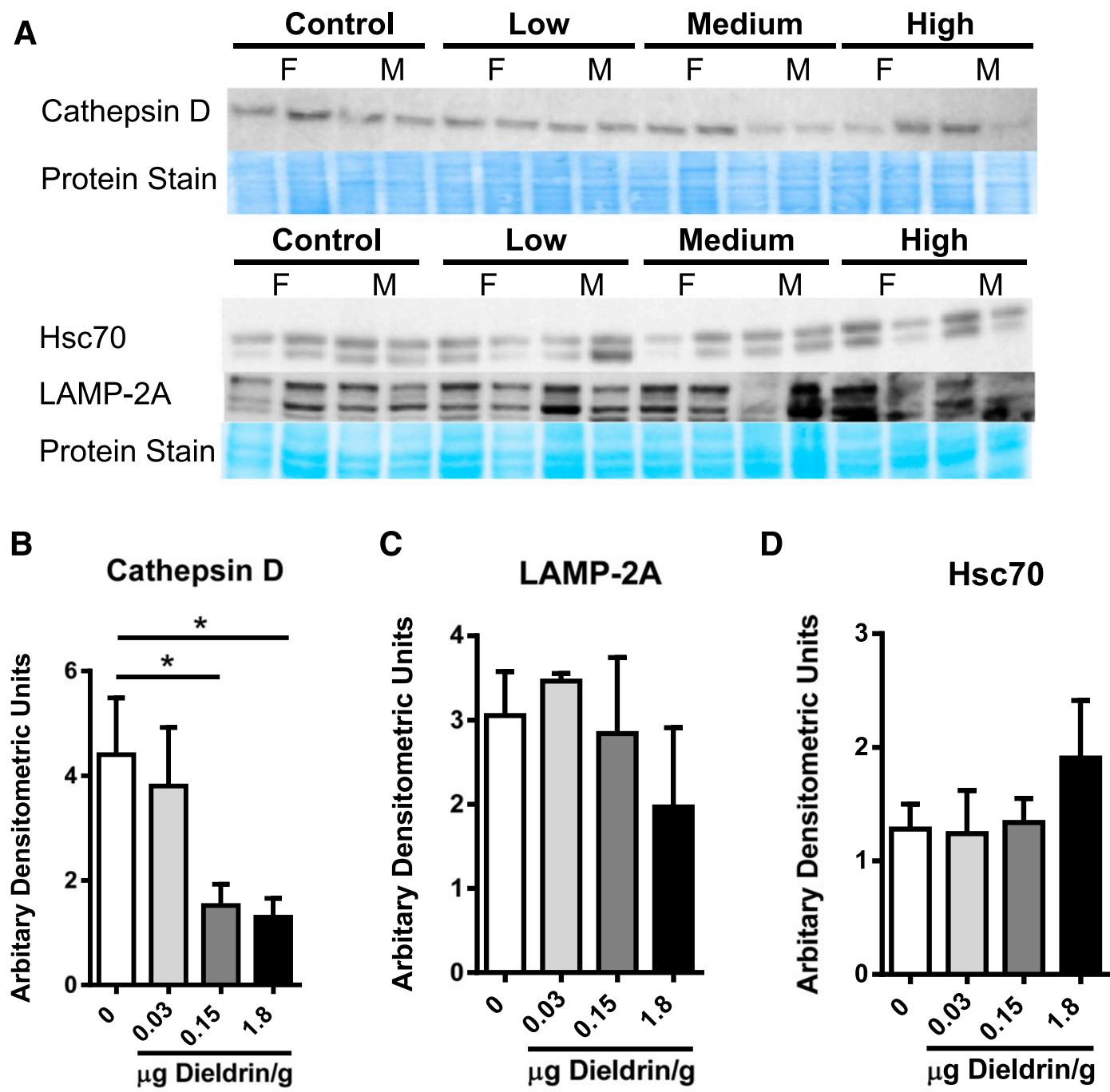

Fig. 6. Immunoblot analysis of autophagy proteins in pooled zebrafish hearts treated with four different concentrations of dieldrin (control, 0.03 , 0.15 , and $1.8 \mu \mathrm{g} / \mathrm{g}$ in feed) for 21 days. ${ }^{*} P<0.05$. (A) Immunoblot images of protein targets in the four different concentrations, along with protein stain loading control separated by treatment group and sex. F, female; M, male. (B) Cathepsin D immunoblot quantification after correction to protein stain. (C) LAMP-2A immunoblot quantification after correction to protein stain. (D) hsc70 immunoblot quantification after correction to protein stain; graphs represent mean densitometric units plus S.E.M. $n=4$ per treatment group.

protein degradation (Yoshimori et al., 1991). Further supporting this hypothesis is our finding that moln 1 and $m \operatorname{coln} 3$ are downregulated by dieldrin treatment. Loss-of-function mutations in MCOLN1 result in mucolipidosis type IV, which is characterized by a buildup of lysosomes containing lipids (Chen et al., 1998), and increased lysosomal lumen $\mathrm{pH}$ has also been identified (Bach et al., 1999); whereas, MCOLN3 inhibition results in defects in lysosomal acidification and impaired autophagosome maturation (Lelouvier and Puertollano, 2011). Furthermore, our microarray data revealed that microtubule-associated proteins 1 and LC3A/B, which are necessary for proper autophagosome maturation (Tanida, 2011), are downregulated in the heart by dieldrin treatment. These data suggest that lysosomal function and cellular trafficking are disrupted by dieldrin treatment. There is only one other report (Kohli et al., 1977) on the effect of dieldrin on lysosome function, which suggests that dieldrin treatment in rat livers upregulates protease activity.

Given that microarray analysis highlighted several lysosomal proteins with reduced expression, we examined the
mTOR/Akt axis, which is known to inhibit lysosomal autophagy. Indeed, mTOR and AKT signaling were upregulated in dieldrin-treated hearts. Previous reports have also described dieldrin as an Akt activator that is mediated by the activation of the G-protein-coupled estrogen receptor (Briz et al., 2011). The mTOR pathway acts as a negative regulator of lysosomal biogenesis and autophagy gene expression through inactivation of transcription factor EB by phosphorylation, preventing its translocation into the nucleus (Roczniak-Ferguson et al., 2012). Recent studies show that autophagy provides protection to the heart by clearing toxic protein aggregates that accumulate in the cell during cardiac stress (Martinet et al., 2009). Our data show that dieldrin blocks this process, potentially contributing to cardiac injury.

In summary, using a zebrafish model, we characterized cardiac-specific pathologic signaling of dieldrin. Underlying molecular changes in dieldrin-exposed hearts include inflammation and disruption of lysosomal signaling. Furthermore, dieldrin regulates genes that are related to the development of 
cardiac arrest and VF, among many other disease associations. Future work should aim to characterize the cellautonomous effect of dieldrin specifically using isolated cardiomyocytes to determine the direct action of dieldrin on the cardiac cells and its physiologic impact.

\section{Authorship Contributions}

Participated in Research Design: Slade, Martyniuk, and Pulinilkunnil Conducted experiments: Slade and Cowie

Contributed new reagents or analytic tools: Martyniuk, Kienesberger, and Pulinilkunnil

Performed data analysis: Slade, Cowie, Martyniuk and Pulinilkunnil

Wrote or contributed to the writing of the manuscript: Slade, Cowie, Martyniuk, Kienesberger, and Pulinilkunnil

\section{References}

Alavanja MC, Hoppin JA, and Kamel F (2004) Health effects of chronic pesticide exposure: cancer and neurotoxicity. Annu Rev Public Health 25:155-197.

Ananya R, Subeena S, Kumar DA, Kumar DT, and Kumar MS (2005) Oxidative stress and histopathological changes in the heart following oral lindane (gamma hexachlorohexane) administration in rats. Med Sci Monit 11:BR325-BR329.

Bach G, Chen C-S, and Pagano RE (1999) Elevated lysosomal pH in mucolipidosis type IV cells. Clin Chim Acta 280:173-179.

Baltazar MT, Dinis-Oliveira RJ, de Lourdes Bastos M, Tsatsakis AM, Duarte JA, and Carvalho F (2014) Pesticides exposure as etiological factors of Parkinson's disease and other neurodegenerative diseases-a mechanistic approach. Toxicol Lett 230:85-103.

Bandala ER, Andres-Octaviano J, Pastrana P, and Torres LG (2006) Removal of aldrin, dieldrin, heptachlor, and heptachlor epoxide using activated carbon and/or Pseudomonas fluorescens free cell cultures. J Environ Sci Health B $\mathbf{4 1}$ $553-569$

Bartlett JJ, Trivedi PC, Yeung P, Kienesberger PC, and Pulinilkunnil T (2016) Doxorubicin impairs cardiomyocyte viability by suppressing transcription factor EB expression and disrupting autophagy. Biochem J 473:3769-3789.

Briz V, Molina-Molina J-M, Sánchez-Redondo S, Fernández MF, Grimalt JO, Olea N, Rodríguez-Farré E, and Suñol C (2011) Differential estrogenic effects of the persistent organochlorine pesticides dieldrin, endosulfan, and lindane in primary neuronal cultures. Toxicol Sci 120:413-427.

Brock JW, Melnyk LJ, Caudill SP, Needham LL, and Bond AE (1998) Serum levels of several organochlorine pesticides in farmers correspond with dietary exposure and local use history. Toxicol Ind Health 14:275-289.

Brugada R, Hong K, Dumaine R, Cordeiro J, Gaita F, Borggrefe M, Menendez TM, Brugada J, Pollevick GD, Wolpert C, et al. (2004) Sudden death associated with short-QT syndrome linked to mutations in HERG. Circulation 109:30-35.

Chen C-S, Bach G, and Pagano RE (1998) Abnormal transport along the lysosomal pathway in mucolipidosis, type IV disease. Proc Natl Acad Sci USA 95:6373-6378

Cowie AM, Sarty KI, Mercer A, Koh J, Kidd KA, and Martyniuk CJ (2017) Molecular networks related to the immune system and mitochondria are targets for the pesticide dieldrin in the zebrafish (Danio rerio) central nervous system. $J$ Proteomics 157:71-82.

Cupul-Uicab LA, Klebanoff MA, Brock JW, and Longnecker MP (2013) Prenatal exposure to persistent organochlorines and childhood obesity in the US collaborative perinatal project. Environ Health Perspect 121:1103-1109.

Fleming L, Mann JB, Bean J, Briggle T, and Sanchez-Ramos JR (1994) Parkinson's disease and brain levels of organochlorine pesticides. Ann Neurol 36:100-103.

Forgac M (2007) Vacuolar ATPases: rotary proton pumps in physiology and pathophysiology. Nat Rev Mol Cell Biol 8:917-929.

Frantz S, Fraccarollo D, Wagner H, Behr TM, Jung P, Angermann CE, Ertl G, and Bauersachs J (2003) Sustained activation of nuclear factor kappa B and activator protein 1 in chronic heart failure. Cardiovasc Res 57:749-756.

Fujii Y, Ito Y, Harada KH, Hitomi T, Koizumi A, and Haraguchi K (2012) Comparative survey of levels of chlorinated cyclodiene pesticides in breast milk from some cities of China, Korea and Japan. Chemosphere 89:452-457.

Gorini F, Chiappa E, Gargani L, and Picano E (2014) Potential effects of environmental chemical contamination in congenital heart disease. Pediatr Cardiol 35 559-568.

Herzig TC, Jobe SM, Aoki H, Molkentin JD, Cowley AW, Jr, Izumo S, and Markham BE (1997) Angiotensin II type1a receptor gene expression in the heart: AP-1 and GATA-4 participate in the response to pressure overload. Proc Natl Acad Sci USA 94:7543-7548.

Hong K, Bjerregaard P, Gussak I, and Brugada R (2005) Short QT syndrome and atrial fibrillation caused by mutation in KCNH2. $J$ Cardiovasc Electrophysiol 16 394-396.

Howe K, Clark MD, Torroja CF, Torrance J, Berthelot C, Muffato M, Collins JE, Humphray S, McLaren K, Matthews L, et al. (2013) The zebrafish reference genome sequence and its relationship to the human genome. Nature 496:498-503.

Hung D-Z, Yang H-J, Li Y-F, Lin C-L, Chang S-Y, Sung F-C, and Tai SCW (2015) The long-term effects of organophosphates poisoning as a risk factor of CVDs: a nationwide population-based cohort study. PLoS One 10:e137632

Jopling C, Sleep E, Raya M, Martí M, Raya A, and Izpisúa Belmonte JC (2010) Zebrafish heart regeneration occurs by cardiomyocyte dedifferentiation and proliferation. Nature 464:606-609.
Jorgenson JL (2001) Aldrin and dieldrin: a review of research on their production, environmental deposition and fate, bioaccumulation, toxicology, and epidemiology in the United States. Environ Health Perspect 109 (Suppl 1):113-139.

Jung CH, Ro S-H, Cao J, Otto NM, and Kim D-H (2010) mTOR regulation of autophagy. FEBS Lett 584:1287-1295.

Karin M, Liu Zg, and Zandi E (1997) AP-1 function and regulation. Curr Opin Cell Biol 9:240-246.

Kiewe P, Gueller S, Komor M, Stroux A, Thiel E, and Hofmann W-K (2009) Prediction of qualitative outcome of oligonucleotide microarray hybridization by measurement of RNA integrity using the 2100 Bioanalyzer capillary electrophoresis system. Ann Hematol 88:1177-1183.

Kim S-A, Kim K-S, Lee Y-M, Jacobs DR, and Lee D-H (2015) Associations of organochlorine pesticides and polychlorinated biphenyls with total, cardiovascular, and cancer mortality in elders with differing fat mass. Environ Res 138:1-7.

Kitazawa M, Anantharam V, and Kanthasamy AG (2003) Dieldrin induces apoptosis by promoting caspase-3-dependent proteolytic cleavage of protein kinase Cdelta in dopaminergic cells: relevance to oxidative stress and dopaminergic degeneration. Neuroscience 119:945-964.

Kohli KK, Siddiqui FA, and Venkitasubramanian TA (1977) Effect of dieldrin on the stability of lysosomes in the rat liver. Bull Environ Contam Toxicol 18:617-623.

Lee D-H, Porta M, Jacobs DR, Jr, and Vandenberg LN (2014) Chlorinated persistent organic pollutants, obesity, and type 2 diabetes. Endocr Rev 35:557-601.

Lee D-H, Steffes MW, Sjödin A, Jones RS, Needham LL, and Jacobs DR, Jr (2011) Low dose organochlorine pesticides and polychlorinated biphenyls predict obesity, dyslipidemia, and insulin resistance among people free of diabetes. PLoS One 6: e15977.

Lelouvier B and Puertollano R (2011) Mucolipin-3 regulates luminal calcium, acidification, and membrane fusion in the endosomal pathway. $J$ Biol Chem $\mathbf{2 8 6}$ 9826-9832.

Lieschke GJ and Currie PD (2007) Animal models of human disease: zebrafish swim into view. Nat Rev Genet 8:353-367.

Liu Y, Asnani A, Zou L, Bentley VL, Yu M, Wang Y, Dellaire G, Sarkar KS, Dai M, Chen $\mathrm{HH}$, et al. (2014) Visnagin protects against doxorubicin-induced cardiomyopathy through modulation of mitochondrial malate dehydrogenase. Sci Transt Med 6:266ra170.

Ljunggren SA, Helmfrid I, Salihovic S, van Bavel B, Wingren G, Lindahl M, and Karlsson H (2014) Persistent organic pollutants distribution in lipoprotein fractions in relation to cardiovascular disease and cancer. Environ Int 65: 93-99.

Martinet W, Agostinis P, Vanhoecke B, Dewaele M, and De Meyer GR (2009) Autophagy in disease: a double-edged sword with therapeutic potential. Clin Sci (Lond) 116:697-712.

Martyniuk CJ, Doperalski NJ, Kroll KJ, Barber DS, and Denslow ND (2013) Sexually dimorphic transcriptomic responses in the teleostean hypothalamus: a case study with the organochlorine pesticide dieldrin. Neurotoxicology 34:105-117.

Martyniuk CJ, Feswick A, Spade DJ, Kroll KJ, Barber DS, and Denslow ND (2010a) Effects of acute dieldrin exposure on neurotransmitters and global gene transcription in largemouth bass (Micropterus salmoides) hypothalamus. Neurotoxicology $\mathbf{3 1}$ 356-366.

Martyniuk CJ, Kroll KJ, Doperalski NJ, Barber DS, and Denslow ND (2010b) Genomic and proteomic responses to environmentally relevant exposures to dieldrin: indicators of neurodegeneration? Toxicol Sci 117:190-199.

McGrath P and Li C-Q (2008) Zebrafish: a predictive model for assessing druginduced toxicity. Drug Discov Today 13:394-401.

Medeiros-Domingo A, Tan B-H, Crotti L, Tester DJ, Eckhardt L, Cuoretti A, Kroboth SL, Song C, Zhou Q, Kopp D, et al. (2010) Gain-of-function mutation, S422L, in the KCNJ8-encoded cardiac K(ATP) channel Kir6.1 as a pathogenic substrate for J wave syndromes. Heart Rhythm 7:1466-1471.

Mehrotra BD, Moorthy KS, Reddy SR, and Desaiah D (1989) Effects of cyclodiene compounds on calcium pump activity in rat brain and heart. Toxicology 54: $17-29$

Milan DJ, Jones IL, Ellinor PT, and MacRae CA (2006) In vivo recording of adult zebrafish electrocardiogram and assessment of drug-induced QT prolongation. Am J Physiol Heart Circ Physiol 291:H269-H273.

Min JY, Cho JS, Lee KJ, Park JB, Park SG, Kim JY, and Min KB (2011) Potential role for organochlorine pesticides in the prevalence of peripheral arterial diseases in obese persons: results from the National Health and Nutrition Examination Survey 1999-2004. Atherosclerosis 218:200-206.

Moore-Carrasco R, García-Martínez C, Busquets S, Ametller E, Barreiro E, LópezSoriano FJ, and Argilés JM (2006) The AP-1/CJUN signaling cascade is involved in muscle differentiation: implications in muscle wasting during cancer cachexia. FEBS Lett 580:691-696.

Moore MN, Allen JI, and Somerfield PJ (2006) Autophagy: role in surviving environmental stress. Mar Environ Res 62 (Suppl):S420-S425.

Nikitin A, Egorov S, Daraselia N, and Mazo I (2003) Pathway studio-the analysis and navigation of molecular networks. Bioinformatics 19:2155-2157.

Ozmen O (2013) Cardiotoxicity and apoptotic activity in subacute endosulfan toxicity and the protective effect of vitamin $\mathrm{C}$ in rabbits: a pathological study. J Environ Pathol Toxicol Oncol 32:53-58.

Pelletier M, Roberge CJ, Gauthier M, Vandal K, Tessier PA, and Girard D (2001) Activation of human neutrophils in vitro and dieldrin-induced neutrophilic inflammation in vivo. J Leukoc Biol 70:367-373.

Roczniak-Ferguson A, Petit CS, Froehlich F, Qian S, Ky J, Angarola B, Walther TC, and Ferguson SM (2012) The transcription factor TFEB links mTORC1 signaling to transcriptional control of lysosome homeostasis. Sci Signal 5:ra42.

Sergeev AV and Carpenter DO (2005) Hospitalization rates for coronary heart disease in relation to residence near areas contaminated with persistent organic pollutants and other pollutants. Environ Health Perspect 113 $756-761$. 
Shaulian E and Karin M (2002) AP-1 as a regulator of cell life and death. Nat Cell Biol 4:E131-E136.

Singh N, Chhillar N, Banerjee B, Bala K, Basu M, and Mustafa M (2013) Organochlorine pesticide levels and risk of Alzheimer's disease in north Indian population. Hum Exp Toxicol 32:24-30.

Singh NK, Chhillar N, Banerjee BD, Bala K, Mukherjee AK, Mustafa MD, and Mitrabasu (2012) Gene-environment interaction in Alzheimer's disease. Am J Alzheimers Dis Other Demen 27:496-503.

Sun F, Anantharam V, Latchoumycandane C, Kanthasamy A, and Kanthasamy AG (2005) Dieldrin induces ubiquitin-proteasome dysfunction in $\alpha$-synuclein overexpressing dopaminergic neuronal cells and enhances susceptibility to apoptotic cell death. J Pharmacol Exp Ther 315:69-79.

Tanida I (2011) Autophagosome formation and molecular mechanism of autophagy. Antioxid Redox Signal 14:2201-2214.

Tsuburaya R, Yasuda S, Ito Y, Shiroto T, Gao JY, Ito K, and Shimokawa H (2011) Eicosapentaenoic acid reduces ischemic ventricular fibrillation via altering monophasic action potential in pigs. J Mol Cell Cardiol 51:329-336. van Wamel AJ, Ruwhof C, van der Valk-Kokshoom LE, Schrier PI, and van der Laarse A (2001) The role of angiotensin II, endothelin-1 and transforming growth factor- $\beta$ as autocrine/paracrine mediators of stretch-induced cardiomyocyte hypertrophy. Mol Cell Biochem 218:113-124.

Weisskopf MG, Knekt P, O'Reilly EJ, Lyytinen J, Reunanen A, Laden F, Altshul L, and Ascherio A (2010) Persistent organochlorine pesticides in serum and risk of Parkinson disease. Neurology 74:1055-1061.

Yoshimori T, Yamamoto A, Moriyama Y, Futai M, and Tashiro Y (1991) Bafilomycin A1, a specific inhibitor of vacuolar-type $\mathrm{H}(+)$-ATPase, inhibits acidification and protein degradation in lysosomes of cultured cells. J Biol Chem 266:17707-17712.

Address correspondence to: Thomas Pulinilkunnil, Department of Biochemistry and Molecular Biology, Dalhousie University, Dalhousie Medicine New Brunswick (DMNB), 100 Tucker Park Road, PO Box 5050, Saint John, NB E2L 4L5, Canada. E-mail: tpulinil@dal.ca 